\title{
Notizen über den gegenwärtigen Stand der Krebsforschung in London und Paris.
}

Briefliche Mitteilungen an das Komitee für Krebsforschung.

Von

\section{Aschoff,}

Göttingen.

Ein mehrmonatlicher Aufenthalt in London und Paris gab mir im letzten Winter Gelegenheit, über den Stand der Krebsforschung in diesen beiden Städten einige Notizen zu sammeln, die ich hiermit dem deutschen Komitee für Krebsforschung übersende.

Seit der bekannten Krebsnummer im Practitioner, April 1899, deren Publikation im wesentlichen das Verdienst des Dermatologen Malcolm Morris, des Herausgebers des Practitioner, ist, hat sich das Interesse an der parasitären und statistischen Erforschnng der Krebskrankheit in England noch erheblich vermehrt und hat seinen beredten Ausdruck in der Gründung eines eigenen Cancer Investigation Committee gefunden, für welches erst noch in jüngster Zeit bedeutende Geldmittel durch private Sammlungen aufgebracht worden sind, auf die ich noch später zu sprechen kommen werde.

Ehe ich auf die Krebsforschung in England selbst eingehe, möchte ich kurz bemerken, dass in den statistischen Mitteilungen der Blaubücher der englischen Kolonien ein recht beachtenswertes Material für die Beantwortung mancher Fragen nach dem Vorkommen bestimmter Krankheiten in Tropengegenden gegeben ist. Um dem deutschen Leser einen Einblick in die Art dieser Statistik zu verschaffen, habe ich die Berichte aus den Regierungskrankenhäusern der englischen Kolonien für das letztveröffentlichte $\mathrm{Jabr}$ zusammengestellt (bereits früher dem Komitee übersandt) und die sich daraus ergebenden Schlüsse auf das Verhältnis von Krebs und Malaria in der Deutschen medizinischen Wochenschrift kurz publiziert.

Durch persönliche Bekanntschaft mit Herrn Dr. Plimmer am St. Mary Hospital in London hatte ich bereits früher Gelegenheit ge- 
habt, seine mikroskopischen Präparate studieren und mich von der Richtigkeit seiner Befunde überzeugen zu können. Die gleichen Gebilde sind ja vor und nach Plimmer von den verschiedensten Autoren gesehen und beschrieben worden. An ihrer Existenz ist nicht zu zweifeln, es fragt sich nur, was sie bedeuten. Während Plimmer selbst an der Hefenatur dieser Gebilde festhält, ist Gaylord zu der Anschauung gelangt, dass es sich um Entwickelungsstadien eines zu den Sporozoen gehörigen Lebewesens handelt. Damit nähert man sich wieder der alten Theorie von der parasitären Bedeutung der Coccidien. Die Plimmerschen Körperchen sind ferner von v. Leyden mit den Kernen der Plasmodiophora brassicae, eines Myxomyceten, verglichen und von einer überraschenden Aehnlichkeit befunden worden. Im Gegensatz zu diesen amerikanischen und deutschen der parasitären Theorie günstigen Deutungen der Plimmerschen Befunde verhalten sich die englischen Pathologen, wie ich mich im persönlichen Gespräch mit Macfadyen, Bulloch, Foulerton, Boyce, Cheatleu. a. überzengen kounte, im ganzen ablehnend gegenüber den von Plimmer verfochtenen Anschauungen ${ }^{1}$ ). Leider war Kollege Plimmer selbst so sehr mit Arbeiten überhäuft, dass er während meines Aufenthaltes in London keine Zeit finden konnte, mir neuere Präparate und neue Beweise für seine parasitäre Theorie zu zeigen. Da ich mich bisher weder in den Sanfeliceschen, noch in den Plimmerschen, noch in den Gaylordschen Präparaten von der Existenz eines durch Einspritzen von Hefekulturen erzeugten Krebses habe überzeugen können, so wäre mir gerade ein derartiger Beweis sehr willkommen gewesen. Doch habe ich, wie gesagt, nach längerem vergeblichen Warten auf eine Demonstration der Plimmerschen Beweisobjekte verzichten müssen. Doch erzählte mir Plimmer, dass or bis in die neueste Zeit hinein positive Resultate bei seinen Hefeziichtungen aus Krebsen erzielt hat. Das reiche Material, welches Plimmer zur Verfügung steht, wird ihm hauptsächlich von dem Cancer Hospital geliefert. Indes ist dieses Hospital keineswegs als dasjenige zu betrachten, welches ausschliesslich oder besonders der Erforschung des Krebses gewidmet ist. Es ist ein relativ kleines Spital, welches hauptsächlich Krebskranke aufnimmt, an welchem aber keine speziellen therapeutischen Versuche etc. vorgenommen werden. Vielmehr werden die Falle chirurgisch behandelt, das operativ entfernte Material Plimmer zur Verfügung gestellt. Genauere Untersuchungen am Sektionsmaterial werden für gewöhnlich nicht vorgenommen.

Das Zentrum für die Krebsuntersuchung bildet vielmehr das

1) Anm. bei der Korrektur. Meine persönliche Anschauung über die Bedeutung dieser Gebilde als Vakuolen habe ich erst kürzlich in einer von Herrn Dr. Spirlas angefertigten Arbeit (Münch. med. Wochenschr., 1903, No. 19) zu begründen versucht. L. As ch off. (Referiert S. 138. Red.) 
Middlesex Hospital, welches eine eigene, ausschliesslich für unheilbare Krebskranke bestimmte Abteilung besitzt, deren klinisches Material zur Prüfung der verschiedensten therapeutischen Verfahren dient. Mit dieser Abteilung ist oin besonderes Laboratorium verbunden, welches ausschliesslich den histologischen, kulturellen und experimentellen Arbeiten über Krebs gewidmet ist. An der Spitze dieser Cancer Research Laboratories steht seit ihrer vor $1 \mathrm{Jahr}$ erfolgten Gründung Dr. Foulerton, dem ich den nachfolgenden Bericht über die historische Entwickelung und augenblickliche Gestaltung der Krebsabteilung am Middlesex Hospital verdanke. Persönlich überzeugte ich mich von dem sehr sauberen und freundlichen Aussehen der Krankensäle und der Gesellschaftszimmer der Kranken, denen jede Hoffnung auf Geresung bis jetzt verschlossen scheint. Denn die mit der Röntgenbehandlung bei verschiedenen Fällen von cancer en cuirasse erzielten sehr auffälligen Besserungen, die sich hauptsächlich in einer. geradezu erstaunlich schnellen Ueberhäutung ausgedehnter Wundflächen äusserten, sind doch nach dem, was ich dank der Freundlichkeit des Kollegen Foulerton persönlich bei den Kranken feststellen konnte, nicht als Heilungen zu bezeichnen. In den Laboratorien wird eifrig an der histologischen Durchforschung des Krebses gearbeitet. Insbesondere war man damit beschäftigt, die Plimmer schen Körperchen von echten Hefen durch besondere Färbungsmethoden zu differenzieren und ihre farbenchemischen Reaktionen noch genauer festzustellen. Foulerton selbst ist mit einer neuen therapeutischen Behandlung des Krebses beschäftigt. Ueber diese und andere Versuche der Krebsabteilung des Middlesex Hospital wird in besonderen Reports ausführlich berichtet werden. Dass jeder Todesfall einer genauen Autopsie mit nachfolgender mikroskopischen Untersuchung unterliegt, will ich als eigentlich selbstverständlich nur kurz erwähnen.

Der Bericht Dr. Foulertons, dessen englisches Original ich beilege, lautet folgendermassen :

\section{Die Laboratorien für Krebsuntersuchung am Middlesex Hospital in London.}

Das für die Untersuchungen nötige Material wird geliefert

1) von den sämtlichen inneren und chirurgischen Stationen des Middlesex Hospital, welche zusammen 320 Betten enthalten;

2) von der speziellen Krebsabteilung, welche 49 Betten enthält (40 für Frauen, 9 für Männer).

I.

Fälle von bösartigen Geschwülsten werden auf die inneren und äusseren Stationen des Middlesex Hospital in gleicher Weise, wie in allen übrigen Londoner Spitälern, aufgenommen. Der Registrator der 
speziellen Krebsabteilung am Middlesex Hospital hat die Pflicht, die klinischen Details, anamnestische Daten und alle sonstigen von dem Direktor des Laboratoriums für Krebsforschung gewünschten Angaben für jeden èinzelnen Fall von bösartigem Tumor, der auf eine innere oder äussere Station aufgenommen wird, zu erheben. Ferner wohnt der Registrator allen Operationen maligner Tumoren bei und hat dafür Sorge zu tragen, dass das gewonnene Material in die Laboratorien abgeliefert wird. Ebenso sind Bestimmungen darüber getroffen, dass das bei etwaigen Sektionen von Krebskranken, die auf den gewöhnlichen Stationen gelegen haben, gewonnene Material den Laboratorien zugestellt wird.

\section{Die besondere Krebsabteilung.}

Diese Krebsabteilung wird durch einen besonderen Fonds unterhalten, der von der Verwaltung des Middlesex Hospital mitverwaltet wird. Die Patienten werden hier von 3 Senior Chirurgen des Middlesex Hospital behandelt.

Diese Krebsabteilung ist bislang die einzige ihrer Art unter allen englischen Spitälern. Ihre Geschichte ist kurz folgende.

Das Middlesex Hospital selbst ist im August 1745 gegrïndet worden. Im Jahre 1791 schenkte Samuel Whitbread dem Spital die Summe von 4000 Pfund mit der ausdrücklichen Bestimmung, dass diese Summe verwendet werden sollte:

.fuir die Behandlung von Krebskranken und die Erforschung dieses Leidens, über welches trotz seiner grossen Verbreitung sowohl in ätiologischer wie auch in therapeutischer Beziehung noch das grösste Dunkel herrsche".

30 Jahre später erhielt das Spital eine zweite grosse Stiftung von einer Dame Alithea Maria Stafford mit der Bestimmung:

"dieselbe für die Behandlung von Krebskranken und für die Erforschung dieser furchtbaren Krankheit zu verwenden".

In den folgenden Zeiten wurden von wohltätigen Personen dem Krebsfonds neue Zuwendungen gemacht, so dass derselbe sich erbeblich vermehrte.

Bis Ende 1899 bildeten die speziellen für Krebsbehandlung bestimmten Säle einen Teil des allgemeinen Krankenhauses und boten für 33 Patienten Raum.

Im Januar 1900 wurden die Frauen der Krebsabteilung in einen besonderen Block, welcher neben dem Hauptgebäude des Krankenhauses errichtet worden war, übergeführt. Dieser neue "Cancer Block“ enthält 3 Säle mit je 12 Betten und 2 kleinere Räume mit je 2 Betten. Ausserdem enthält derselbe noch die Laboratorien, einen Sektionssaal, die Räume für die Administration und für die Schwestern. 


\section{L. Aschoff, Der Stand der Krebsforschung in London und Paris.}

Die Männerabteilung befindet sich vorläufig noch im grossen Hauptgebäude. Sie enthält 9 Betten.

Diese 49 Betten sind ausschliesslich für solche Fälle von malignen Tumoren bestimmt, wo eine Rettung durch chirurgische Eingriffe ausgeschlossen ist. Die Kranken werden dort bis zu ihrem Tode gepflegt. Die einzigen Gründe für Entfernung eines Patienten sind Insubordination oder eintretende Geistesstörung. Auf Wunsch der verschiedenen Stifter des Krebsfonds werden die Patienten etwas besser. als durchschnittlich in den Londoner Spitälern verpflegt, indem ihre Kost abwechslungsreicher gestaltet wird, indem ihnen ferner eigene Gesellschaftsräume (die, wie sich Berichterstatter iiberzeugen kounte, äusserst freundlich und behaglich mit Blumen, Sesseln, kleinen Springbrunnen etc. eingerichtet sind) zur'Verfügung stehen, den Männern das Rauchen gestattet ist etc. Der Besuch der Angehörigen ist jederzeit erlaubt, und für musikalische und andere Unterhaltungen ist ebenfalls Sorge getragen.

Die Kosten des neuen Cancer Block belaufen sich auf 196913 Pfund, von denen 18394 auf die Gebäude, 1299 auf die innere Einrichtung entfallen.

Da die Patienten den ärmeren Klassen der Bevölkerung angehören, so wird selbstverständlich keine Bezahlung von ihnen genommen.

III. Die Laboratorien für Krebsforschung.

Zur selben Zeit, wo der neve "Cancer Block" in Benutzung genommen wurde, begannen auch die wissenschaftlichen Untersuchungen des reichlich vorhandenen Materials. Da zur Zeit keine grösseren Mittel zur Verfügung standen, so waren auch die Einrichtungen der Laboratorien noch etwas beschränkt. Die Laboratorien bestehen aus einem grösseren Raum für den Direktor und zwei kleineren Räumen für histologische und bakteriologische und für chemische Untersuchungen. A. G. R. Foulerton, F. R. C. S., wurde zum Direktor der Krebsabteilung, Dr. Hillier zum Assistenten, Dr. Clifford zum Registrator ernannt.

Der Direktor wurde beauftragt, ein Schema für die vorzunehmenden Untersuchungen aufzustellen. Dasselbe folgt anbei. Die Laboratorien wurden im Februar 1900 eröffnet.

Das Material, welches den Laboratorien geliefert wird, stammt von ca. 250 Fällen maligner Tumoren pro anno. Von der speziellen Krebsabteilung kommen jährlich 70-80 Fälle zur Sektion, die von dem Staff der Krebsabteilung ausgeführt werden. Aus den übrigen Sälen des Hauptkrankenhauses gelangen noch jährlich ca. 20-25 Fälle von Krebs etc. zur Autopsie. In den übrigen 150 Fällen handelt es sich um operativ entfernte Geschwülste. Die Untersuchung eines jeden Tumors (oder Sektionsfalles) wird so vollständig wie möglich vorge- 
nommen. Der Bericht über die ersten 500 Fälle wird im Laufe dieses Sommers veröffentlicht werden. Der Bericht wird auch die spezielleren in den Laboratorien ausgeführten Arbeiten berühren. Solche Berichte sollen alle 2 Jahre veröffentlicht werden.

Die im Middlesex Hospital bestehende Abteilung für Krebsforschung erhält keine staatliche Unterstützung. Sie erhält sich ausschliesslich aus dem Krebsfonds. Der Staat fordert sogar von dem Hospital dieselben Abgaben, wie von einem kommerziellen Unternehmen. Selbst der in den Laboratorien gebrauchte Alkohol muss hoch versteuert werden.

$$
\text { gez, Alex. G. R. Foulerton, }
$$

Direktor.

\section{Schema für die Untersuchungen :}

1) In jedem Falle von Carcinom oder Sarkom, der auf eine innere oder äussere Abteilung oder auf die Krebsabteilung des Middlesex Hospital aufgenommen wird oder dort stirbt, soll eine systematische Untersuchung vorgenommen werden. Dieselbe soll dazu dienen:

a) eine genügende Klassifizierung der Sarkome zu ermöglichen,

b) die Ursache der Carcinome und Sarkome zu ergründen.

A. Während eine genügende histologische Klassifizierung der Krebse seit Jahren existiert, ist dieselbe für die Sarkome noch sehr mangelhaft.

B. Aetiologische Forschung. Die neueren Theorien über die Blastomyceten und ihre Beziehungen zu den Carcinomen erheischen genaue Berücksichtigung, wenn auch die von den italienischen Forschern vorgebrachten Beweise noch keineswegs allgemein acceptiert sind. Auch die bakteriologische Forschung ist notwendig, wenn auch nur, um durch negative Befunde zu beweisen.

Die Protozoentheorie ist jetzt im ganzen von den Pathologen verlassen und bedarf keiner weiteren Verfolgung.

Die ältere Theorie, dass die Krebse, unabhängig von parasitären Einfliissen, aus abnorm verlagerten Epithelresten hervorgehen, scheint die beachtenswerteste zu sein, von der man noch am meisten durch weitere Forschung erwarten kann.

C. Aetiologie des Sarkoms. Unsere Kenntnisse über die Pathologie der Sarkome sind viel ungenügender als diejenigen über die Krebse. Wir haben allen Grund zu der Annahme, dass alle verschiedenen Krebsformen nur Ausdruck ein und desselben krankhaften Vorganges sind. Bei den Sarkomen liegt die Sache anders. Neuere Untersuchungen (Curtis, Busse, N. Biargi) zeigen, dass manche klinisch als Sarkom angesprochene Tumoren nichts anderes als Hefeinfektionen darstellen. Ebenso mögen manche als "Lymphosarkom" 
bezeichnete Geschwülste in Wirklichkeit parasitäre Infektionen darstellen.

Auf der anderen Seite ist kein Grund vorhanden, für alle Sarkome eine parasitäre Ursache anzunehmen. Die Beziehungen der Sarkome zu den chronischen Entzündungen bedürfen weiterer genauer Untersuchung.

D. Eine sorgfältige, auf histologische Untersuchungen gestützte Registrierung aller Sarkom- und Carcinomfälle, welche auch Alter und Geschlecht beriucksichtigt, wird in Verbindung mit der allgemeinen Gesundbeitsstatistik von grossem Werte sein. In den Generalregistern werden bis jetzt Krebse und Sarkome unter den Begriff „Cancer" zusammengefasst, so dass eine Statistik über die Häufigkeitsverhältnisse zwischen Carcinom und Sarkom unmöglich ist. Eine genaue Trennung von Carcinom und Sarkom wird daher auch mehr Licht auf die bisher unter der Rubrik „Krebs" gegebenen Erkrankungen in den Registrar-General's Returns werfen.

2) Systematische Blutuntersuchungen (Färbung und Zählung der Leukocyten) in ausgewählten Fällen von malignen Tumoren, vor und nach der Operation, und in unoperierten Fällen werden vielleicht weitere Anhaltspunkte auch für die Diagnose dieser Krankheiten ergeben.

3) Quantitative Harnuntersuchungen etc. werden in den chemischen Laboratorien auszuführen sein.

Als ich London verliess, erzählte mir bereits Dr. Foulerton, dass man von seiten der Royal Colleges of Surgeons and Physicians die Frage der Krebsforschung in grösserem Umfange aufnehmen wollte, und dass man zunächst an die Sammlung eines grösseren Fonds dächte, um verschiedene geplante Arbeiten auszuführen. Am 23. Okt. 1901 brachte Henry Morris, Vizepräsident des Royal College of Surgeons, die Angelegenheit in einer gemeinschaftlichen Sitzung der Präsidien der beiden Colleges, des Finanzkomitees derselben und des Laboratoriumkomitees (welchem die Aufsicht über die von den beiden Colleges gegründeten Laboratorien für experimentelle Medizin zufällt) zur Sprache. Man hoffte damals auf das Zustandekommen einer grösseren wohltätigen Stiftung und glaubte daher an die Ausführung folgenden Beschlusses baldigst herantreten zu können: „Die anwesenden Mitglieder der vereinigten Präsidien etc. billigen den Vorschlag, grössere Geldsummen zu sammeln, die der Erforschung der Ursache, der Verhütung und Heilung der Krebskrankheit dienen sollen. Unter der Kontrolle eines geeigneten Komitees soll ein Schema für diese Krebsforschung ausgearbeitet werden und es wird in den Laboratorien der Royal Colleges leicht ein Platz für die nötigen Untersuchungen einzurichten sein." 
Das im Laufe der letzten Monate ausgearbeitete vorläufige Schema ist nm in der British Medical Journal Nummer vom 25. Jan. d. J. publiziert worden.

Ich verweise auf diesen Auszug, möchte nur kurz betonen, dass von den geplanten Unterstützungen der verschiedenen Formen von Krebsforschung der weitere Ausbau der Krebslaboratorien am Middlesex Hospital zunächst die grösste Aussicht auf Verwirklichung hat, und dass diese Laboratorien auch in Zukunft den Mittelpunkt der Krebsforschung bilden werden. Ein Teil der Gelder wird zur Unterstiutzung der Plimmerschen Arbeiten am St. Mary Hospital bezw. an dem unter A. Macfadyens Leitung stehenden Jenner-Institut verwendet werden.

Das neue ins Leben tretende "Cancer Investigation Committee" wird bestehen:

1) aus 5 Kuratoren (zur Verwaltung der Gelder),

2 dem Präsidenten und Censor des R. College of Physicians,

3) dem Präsidenten und 2 Vizepräsidenten des R. College of Surgeons,

4) den Mitgliedern des Laboratoriumkomitees der R. Colleges,

5) einem von dem Local Gouvernement ernannten Mitgliede.

Daneben wird ein aus Mitgliedern des Laboratoriumkomitees der Royal Colleges und aus Mitgliedern des R. Colleges selbst bestehendes Advisory-Board errichtet werden, das dem Generalkomitee Vorschläge in verschiedenster Richtung zu machen hat. Unter diesen möchte ich nur den einen Punkt hervorheben: The establishement of a system of correspondence with foreign and colonial laboratories and workers.

Es liegt also ohne Frage auch in England ein Bedürfnis vor, mit den auswärtigen Komitees für Krebsforschung in Verbindung zu treten. In persönlicher Aussprache hat mir Dr. Foulerton, Direktor der Krebslaboratorien am Middlesex Hospital und Sekretär des Krebskomitees in London, seine Bereitwilligkeit. erklärt, in jeder Beziehung dem deutschen Komitee zu Diensten zu sein und mit ihm gemeinschaftlich zu arbeiten. Ich lege zwei Briefe des Herrn Dr. Foulerton, welche diese Bereitwilligkeit noch einmal ausprechen, dem Berichte desselben bei.

Ich erlaube mir schliesslich den Vorschlag sowohl

Herrn Alexander G. K. Foulerton, F. R. C. S.,

Director of the Cancer Research Laboratories

Secretary for Scientific Purposes to the Cancer Investigation Committee, the Middlesex Hospital, London W., 
wie auch

Herrn Henry Morris, F. R. C. S.,

Senior Surgeon to the Middlesex Hospital,

Chairman of the Cancer Investigation Committee,

8 Cavendish Square, London W.,

der sich um die Entwickelung des Krebskomittees die Hauptverdienste erworben hat,

zu auswärtigen Mitgliedern zu ernennen.

Herr Dr. Plimmer ist ja bereits, soviel ich weiss, auswärtiges Mitglied. Herr Dr. Henry Morris ist nicht mit Malmoolm Morris, dem Herausgeber des Practitioner zu verwechseln. Letzteren habe ich leider nur flüchtig kennen gelernt, aber die von ihm redigierte Krebsnummer des Practitioner beweist das grosse Interesse, das auch dieser Kollege für die Krebsfrage besitzt. Da ich seine persönlichen Intentionen nicht kenne, kann ich mir keinen Vorschlag in dieser Richtung erlauben.

Schliesslich erwähne ich noch, dass sich 19 Ampton-Street, London W C, ein Cancer Statistic Bureau befindet, über dessen Bedeutung ich aber nichts erfahren konnte.

Nachtrag bei der Korrektur: Ueber den weiteren Ausbau des Komitees für Krebsforschung in London hat die medizinische Presse Englands, besonders Lancet und British Medical Journal, vorlänfige Mitteilungen gebracht.

Danach ist bereits ein beträchtlicher Cancer Research Fund gesammelt worden.

An der Spitze der Krebsforschungs-Stiftung steht ein Präsidium, dessen erster Präsident der Prinz von Wales ist. Ihm steht ein Generalkomitee, ein Exekutivkomitee und endlich der wissenschaftliche Vorstand des zu gründenden Institutes für Krebsforschung zur Seite. Als Generaldirektor dieses Institutes ist der durch seine Arbeiten aus dem Ehrlichschen Institut bereits bekannte Kollege E. F. Bashford gewählt worden.

$\mathrm{Zu}$ dem Exekutivkomitee gehören u. a. Henry Morris und M a cf a dyen, der Direktor der tierärztlichen Hochschule. Von einer Unterstützung der schon bestehenden Laboratcrien für Krebsforschung am Middlesex Hospital ist vorläufig Abstand genommen worden.

Ein offizieller Bericht des Komitees ist bis jetzt, soweit ich weiss, nicht erschienen ${ }^{1}$ ). Dagegen liegt der 1. Band der Reports from the Cancer Research Laboratories of the Middlesex Hospital vor. Er enthält Arbeiten von $\mathrm{Hen} \mathbf{r} \mathrm{y}$ W. P. Young über die pathogenen Wirkungen der Blastomyceten, von Cecil Price-Jones über Blutveränderungen bei Erkrankung an bösartigen Geschwülsten, von W. T. Hillier über statistische Erhebungen bei 500 Krebsfällen, von A.

1) Ist inzwischen erschienen (Red.). 
Foulerton und Cecil Price-Jones über bakteriologische Untersuchungen in Krebs- und Sarkomfällen.

Auch in Frankreich hat man sich in den letzten Jahren eifriger mit der Krebsfrage, besonders in therapeutischer Hinsicht, beschäftigt: Dagegen sind die statistischen Arbeiten noch gering an Zahl und liefern keine genügende Basis, um ein Urteil über die Zunahme des Krebses, epidemisches Auftreten etc. zu fällen, obwohl die Arbeiten von Arnaudet, Brunon, Niquet über den Krebs in der Normandie in diesem Sinne gedeutet worden sind. Wlaeff und Trifon off führen das häufige Auftreten des Krebses in der Normandie auf die reichliche Konsumierung zu frischen Käses und verdorbener Früchte zurïck. Ueber Krebshänser und über natürliche und künstliche Uebertragungen des Krebses ist mehrfach berichtet. Die genaueren Angaben finden sich bei $\mathrm{Tr}$ ifon off, L'étiologie et la sérothérapie des tumeurs malignes, Paris 1901. Trifonoff hat auch die Literatur iiber Serotherapie des Krebses, der man in Frankreich besondere Aufmerksamkeit gewidmet hat, zusammengestellt. Trifon off bespricht zunächst die verschiedenen Theorien über die parasitäre Aetiologie des Krebses und schliesst sich voll und ganz der in Frankreich besonders von W l a eff vertretenen Anschaunng über die ursächlichen Beziehungen zwischen Hefepilzen und malignen Tumoren an. Die in jüngster Zeit von Borrel gegen alle als parasitäre Gebilde in Krebszellen gedenteten Einschlïsse erhobenen Einwände (Annales de l'Inst. Pasteur fév. 1901), dass dieselben, wie schon von den verschiedensten deutschen und italienischen Forschern betont worden ist, nur Degenerationsprodukte der Kerne, des Protoplasmas seien und dass die eigenartigen, an Amöbenkerne erinnernden, mit zentralem Kern versehenen runden Körperchen, wie sie Plimmer zuerst eingehend beschrieben und abgebildet hat, auf Degeneration der Zentrosomen zurückzuführen seien, weil man die gleichen Bilder bei der Spermatogenese im Hoden der Nager beobachtet, werden von Trifonoff mit wenigen Worten abgelehnt. Dass man mit Hefepilzen Granulome erzengen kann, ist für Trifon off eine genügende Stütze, um die aus Krebsen gezüchteten Hefen als die Erreger des Krebses anzusprechen Die ausländische Literatur ist übrigens dem Verfasser gut bekannt, wenn auch einige Irrtümer unterlaufen. Auf der angeblich bewiesenen Tatsache, dass Hefepilze die bösartigen Geschwülste erzeugen, beruht die neue Serotherapie in Frankreich. Sie gewann um so leichteren Boden, als alle früheren Versuche, den Krebs durch Injektion von Bakterienprodukten und Bakterien selbst (Erysipelkokken) zu heilen, auch in Frankreich fehlgeschlagen waren. Dass auch bei dieser Behandlung gelegentlich auffallende Besserungen vorkommen, ist bekannt. Kollege Marmorek teilte mir persönlich mit, dass er unter 12 Fällen, die 
er mit Streptokokken behandelt hat, 2 mal überraschende Resultate erhielt, indem bei einem cancer en cuirasse und bei einem Zungenund Magenkrebs ein vollständiger Stillstand des Krebswachstums und eine narbige Umwandlung des Krebsgewebes eintrat.

Die ersten Versuche, durch direkte Injektionen mit abgetöteten Hefekulturen den Krebs zu heilen, stellte de Baker in Paris an. Indes liegen keine wissenschaftlich ernst zu nehmenden Publikationen über die mit dieser Methode gewonnenen Resultate vor. Einen anderen Weg beschritt W la eff $f$, der zunächst Versuche über die Agglutinierung: der Hefezellen durch ein Antihefeserum anstellte. Diese Versuche fielen, wie das auch von anderen Forschern (M a c fa dy e $\mathrm{n}$ ) festgestellt worden ist, positiv aus. Wenn nun die Hefe wirklich die Krebse erzengt, so miisste man auch mit Hülfe eines solchen Serums heilend auf Krebsgeschwülste einwirken können. Die Wla e ffschen Versuche, entzündliche Folgen der Hefeinjektion bei Tieren durch Einspritzung seines Immunserums zu verhindern, sollen glejchfalls günstige Resultate ergeben haben. Ferner will Wlaeff bei seinen gleichzeitigen Injektionen von Hefe und Serum Modifikation des Entziundungsprozesses in der Bauchhöhle seiner Versuchstiere beobachtet haben, die er als Adenome des Pankreas, der Leber, des Dickdarmes bezeichnet.

Wla eff behandelte nun mit diesem Serum, welche er durch Hefeeinspritzungen bei Enten und anderen Tieren erhalten hatte, krebskranke Menschen. Diese Behandlungen fanden vor allem im Hospital Lariboisière auf der Abteilung des Prof. Reynier und im Hospital Beaujon auf der Abteilung des Prof. Berger statt. Ueber die Resultate dieser Behandlungen hat Wla eff mehrfach berichtet, und Trifon off hat dieselben noch einmal in seiner Abbandlung zusammengefasst.

Es ist nicht nötig, zu betonen, dass von einer positiven Heilung keine Rede ist. Es handelt sich in allen nicht operierten Fällen nur um vorübergehende Besserungen. Oft ist die Injektion nur als Unterstützung bei der Operation herangezogen worden; der Heilerfolg ist natürlich der operativen Entfernung des Tumors zuzuschreiben. In anderen Fällen fehlt die mikroskopische Bestätigung der Diagnose Krebs, so dass das wissenschaftliche Ergebnis der Trif o n of fschen Publikation als recht gering zu bezeichnen ist. Um mich persönlich von den im Hospital Lariboisière erzielten Resultaten zu iiberzengen, besuchte ich dasselbe in Gemeinschaft mit Herrn Kollegen M e yer von der L e y d en schen Klinik. Leider hörten wir, dass Dr. W la eff bereits seit einigen Monaten Paris verlassen hat, da er seine Tierexperimente wegen fehlender Mittel hätte aufgeben müssen. Von einer Behandlung der Krebskranken mit Wla effschem Serum war keine Rede mehr.

Ebensowenig Erfolg haben die Versuche von Richet und Héri- 
court gehabt, welche Esel mit Krebsbrei behandelten und das Serum derselben bei Krebskranken injizierten. Bra glaubt, in einem Ascomyceten den Erreger des Krebses entdeckt zu haben. Aber auch sein Serum hat keine positiven Resultate ergeben.

Um so begeisterter klingen die Schilderungen die Le Roy von seiner Behandlung des Krebses mit Einspritzungen von Arsen und Chinin in der Januarsitzung der Académie de Médecine gegęben hat. Ich hatte leider keine Gelegenheit, mich persönlich von den Erfolgen dieses therapeutischen Vorgehens zu überzeugen.

Von den neuesten Mitteilungen über die Aetiologie des Krebses seien noch diejenigen von $\mathrm{Bosc}$ in Montpellier und von Mayet in Lyon erwähnt. Bosc will durch Einspritzen von Schafpockenmaterial echte Leberkrebse erzeugt haben. Die kurzen histologischen Berichte, die bis jetzt vorliegen, genügen nicht, um den Leser von der wirklichen Krebsnatur der erzielten Veränderungen zu überzeugen. Ganz unhaltbar sind die Behauptungen von Mayet, der Krebse längere Zeit macerierte, die Macerationsflüssigkeit durch ein Chamberlandfilter filtrierte, das Filtrat Tieren injizierte und dadurch Carcinome erzeugt haben will. Die histologischen Beschreibungen passen genau auf beliebig erzeugte Granulome und lassen jeglichen Anhaltspunkt für die Diagnose Krebs vermissen.

Im erfreulichen Gegensatz zu diesen recht phantastischen Versuchen steht die ruhige Beurteilung, welche die ganze Krebsfrage im Pasteur-Institut findet. Während auf der einen Seite Metschnikoff, Mesnil, Marmorek die Bedeutung der Statistik für das Anwachsen des Krebses und das epidemische oder endemische Vorkommen desselben voll anerkennen und den möglichen parasitären Charakter der Krebskrankheit betonen, finden andererseits die-verschiedenen Veröffentlichungen über die supponierten Krebskeime in Borrel eine gründliche und auf guten histologischen Kenntnissen begrïndete Kritik, die bisher nur zur Abweisung aller Krebsparasiten geführt hat.

Anm. bei der Korr.: In seiner letzterschienenen Arbeit (Ann. de l'Inst. Pasteur, fév. 1903) hat Borrel den erneuten Nachweis geführt, dass auch die bei der Schafpockenkrankheit beobachteten Zelleinschlüsse nicht parasitärer Natur sind. Da er glaubt, dass die Schafpocken durch einen sehr kleinen, in die Gruppe des Erregers der Maul- und Klauenseuche gehörenden Mikroorganismus hervorgerufen werden, und seiner Meinung nach die bei den Schaf-, Kuh- und Menschenpocken eintretenden Veränderungen des Epithels denjenigen beim Carcinom ähnlich, wenn auch nicht mit ihnen identisch sind, so hält er eine parasitäre Entstehung des Carcinoms durch Infektion mit sehr kleinen, filtrierbaren, bisher nicht sichtbar zu machenden Mikroorganismen nicht für ausgeschlossen. 
Wie ich schon eingangs betonte, ist die statistische Arbeit über Krebs in Frankreich noch nicht so weit entwickelt wie in Deutschland, England, Amerika u. s. w. Durch persönliche Bekanntschaft mit Herrn Dr. med. Treille, Prof. honoraire des maladies des pays chauds, Mitglied des französischen Senats, Dezernenten für die die öffentliche Hygiene betreffenden Regierungsvorlagen, exfuhr ich, dass Herr Dr. Treille selbst, der seit langen Jahren die Statistik der Infektionskrankheiten in Frankreich bearbeitet, der Krebsfrage grosses Interesse entgegenbringt und sich schon längere Zeit mit dem Gedanken trägt, auch über den Krebs in Frankreich statistische Erhebungen zu veranstalten. Da Herr Dr. Treille erst kürzlich wieder in der Debatte über die neuen hrgienischen Gesetze im Senat seine grindliche Kenntnis mit statistischen Bearbeitungen dieser Fragen gezeigt hat, so glaube ich, dem deutschen Komitee für Krebsforschung Herrn Dr: Treille als auswärtiges Mitglied neben Herrn Dr. Metschnik off', Herrn Dr. Borrel vorschlagen zu müssen. Herr Dr. Treille wird gern bereit sein, soweit es seine sonstige Arbeit gestattet, das für die Krebsforschung wichtige Material in Frankreich zu sammeh. Ich glaube um so mehr die Wahl des Herrn Dr. Treille empfehlen zn können, als derselbe lange Jahre Regierungsarzt in Algier war und dort bereits dem Vorkommen von Krebs grosses Interesse zugewendet hat, u. a. anch bereits die auffallenden lokalen Differenzen in der Häufigkeit des Krebses, eine sehr geringe Disposition der Eingeborenen für gewisse Krebsarten etc. festgestellt hat. Herr Dr. Treille ist anch gern bereit, durch jungere Kollegen in den Spitalem Algiers genauere Erhebungen iber den Krebs bei Eingeborenen vornehmen zu lassen.

Ich will noch erwähnen, dass das von Duplay vor Jahren ins Leben gerufene, ausschliesslich der Carcinomfrage gewidmete Journal nicht mehr existiert.

Paris, den 28. März 1902. 